\title{
Review of: "The severity of glomerular endothelial cell injury is associated with infiltrating macrophage heterogeneity in endocapillary proliferative glomerulonephritis"
}

\author{
Yohei Ikezumi ${ }^{1}$ \\ 1 Fujita Health University
}

Potential competing interests: The author(s) declared that no potential competing interests exist.

The authors examined relationship between endocapillary proliferation and macrophage infiltration in patients with post-streptococcal acute GN (PSAGN), Henoch-Schönlein purpura nephritis (HSPN), and lupus nephritis (LN). $\square$ They found that the severity of endothelial cell injury was associated with differences in infiltrating macrophage phenotypes.

This is potentially an interesting study with detailed pathological analysis using immunostaining; however, I have some concerns as listed below:

1. Why did the authors examine only the number of M2-phenotype macrophages? Can CD68-positive cells without M2 markers be said to be M1 phenotype?

2. What are the biopsy indications for patients with PSAGN and what was the timing of their biopsy? If their biopsies were done later in the chronic phase, could their pathological findings be closer to those of patients with HSPN or LN? Similarly, if biopsies of HSPN or LN patients were performed earlier, could morphological findings be closer to PSAGN?

3. In the same context as above, could the number or proportion of M2 macrophages be lower if biopsies of PSAGN patients were performed earlier?

Overall, from the author's discussion, it is unclear whether they consider macrophages to be effector cells for endocapillary injury or just markers for assessing the severity of endocapillary injury. Do the authors believe that the same macrophage-mediated mechanism is involved in endocapillary injury in three different diseases? 